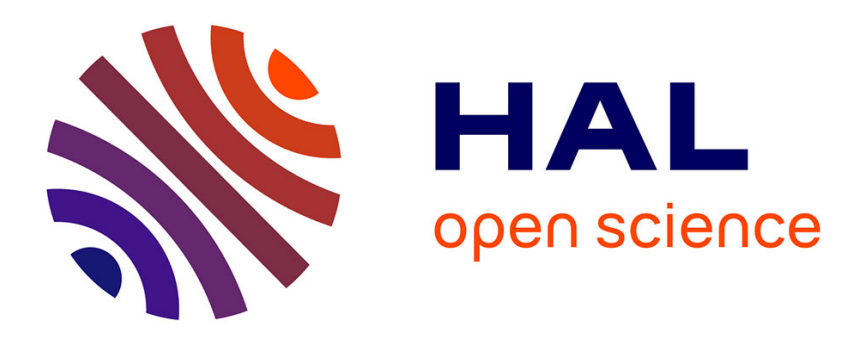

\title{
Étude du schéma de désintégration de 124Sb
}

\author{
J.P. Zirnheld, R. Henck
}

\section{To cite this version:}

J.P. Zirnheld, R. Henck. Étude du schéma de désintégration de 124Sb. Journal de Physique, 1968, 29

(2-3), pp.144-148. 10.1051/jphys:01968002902-3014400 . jpa-00206631

\section{HAL Id: jpa-00206631 https://hal.science/jpa-00206631}

Submitted on 1 Jan 1968

HAL is a multi-disciplinary open access archive for the deposit and dissemination of scientific research documents, whether they are published or not. The documents may come from teaching and research institutions in France or abroad, or from public or private research centers.
L'archive ouverte pluridisciplinaire HAL, est destinée au dépôt et à la diffusion de documents scientifiques de niveau recherche, publiés ou non, émanant des établissements d'enseignement et de recherche français ou étrangers, des laboratoires publics ou privés. 


\title{
ÉTUDE DU SCHÉMA DE DÉSINTÉGRATION DE ${ }^{124}$ Sb
}

\author{
Par J. P. ZIRNHELD, \\ Pile Universitaire de Strasbourg, \\ et R. HENCK, \\ Département de Physique des Rayonnements et d’Électronique Nucléaire, 67-Strasbourg-Cronenbourg.
}

(Reçu le 11 septembre 196\%.)

\begin{abstract}
Résumé. - Le rayonnement $\gamma$ provenant de la décroissance de ${ }^{124} \mathrm{Sb}$ a été étudié à l'aide d'un détecteur de germanium compensé au lithium. La précision en énergie est de l'ordre de $0,5 \mathrm{keV}$. Des mesures de coïncidences $\gamma-\gamma$ ont été faites avec un système lent-rapide de temps de résolution égal à $150 \mathrm{~ns}$, les détecteurs utilisés étant deux diodes au germanium.

La discussion de l'ensemble des résultats a permis de modifier la place de plusieurs transitions dans le schéma de désintégration et d'introduire un niveau supplémentaire à $2773 \mathrm{keV}$.

Abstract. - The $\gamma$-ray spectrum of ${ }^{124} \mathrm{Sb}$ has been re-investigated by means of $\mathrm{Ge}(\mathrm{Li})$ counters. The accuracy obtained in energy measurements is about $0.5 \mathrm{keV}$. The $\gamma-\gamma$ coincidence spectra have been studied with a slow-fast system, utilizing two $\mathrm{Ge}(\mathrm{Li})$ detectors.

The discussion of the results leads us to modify the level scheme and add an additional level at $2773 \mathrm{keV}$.
\end{abstract}

1. Introduction. - Les noyaux pair-pair de masse moyenne $(A \leqslant 150)$ ont déjà fait l'objet de nombreux travaux expérimentaux et théoriques. Divers modèles nucléaires, dérivés de celui de Bohr et Mottelson, ont été développés [1-3] : ils permettent, en général, d'expliquer qualitativement les régularités observées dans un grand nombre de noyaux pair-pair, mais divergent dans la description des spins des états d'énergie plus élevée. L'étude expérimentale des niveaux d'excitation de tels noyaux, comme ${ }^{124} \mathrm{Te}$, fournit ainsi des renseignements intéressants sur la validité des modèles théoriques.

Bien que de nombreux travaux [4 à 11] aient déjà été consacrés à l'examen des niveaux excités de ${ }^{124} \mathrm{Te}$, les spins et parités de plusieurs d'entre eux restent encore inconnus. Par ailleurs, le spectre $\gamma$ obtenu avec un détecteur au germanium nous a montré l'existence de plusieurs transitions nouvelles. C'est pourquoi nous avons entrepris une étude de la désintégration de ${ }^{124} \mathrm{Sb}$ alimentant les niveaux excités de ${ }^{124} \mathrm{Te}$.

2. Méthode expérimentale. - La source utilisée, fournie par le C.E.A., est constituée par ${ }^{124} \mathrm{Sb}$ ( $T=60$ jours), obtenu par capture radiative à partir de ${ }^{123} \mathrm{Sb}$. Elle contient également des traces de ${ }^{122} \mathrm{Sb}$ ( $T=2,8$ jours). Par suite, pour éviter toute influence parasite de cet émetteur, nous n'avons commencé l'étude du spectre $\gamma$ de ${ }^{124} \mathrm{Sb}$ qu'après dix périodes de décroissance de ${ }^{122} \mathrm{Sb}$, soit environ 30 jours.

Pour la détermination des spectres $\gamma$, nous avons utilisé une diode N.I.P. au germanium compensé au lithium $\left(1,2 \mathrm{~cm}^{2}\right.$ de surface, $6 \mathrm{~mm}$ d'épaisseur utile) ayant une résolution de $1,5 \mathrm{keV}$ à basse énergie $\left(222 \mathrm{keV},{ }^{182} \mathrm{Ta}\right)$, grâce à l'utilisation d'un préamplificateur à transistor à effet de champ refroidi.

Des mesures de coïncidences $\gamma-\gamma$ ont été faites avec un système lent-rapide, de temps de résolution égal à $150 \mathrm{~ns}$, les détecteurs $\gamma$ étant deux diodes au germanium de structure coaxiale et de volumes sensibles égaux à 5 et $2 \mathrm{~cm}^{3}$ respectivement.

3. Spectres des rayonnements $\gamma$. - Les spectres $\gamma$ mesurés dans différents domaines d'énergie sont représentés sur les figures 1 à 3 . Les énergies et les intensités relatives des rayonnements $\gamma$ mis en évidence sont indiquées dans le tableau I et comparées avec les résultats des travaux précédents. La variation, en fonction de l'énergie, de l'efficacité intrinsèque de détection par effet photoélectrique de la jonction utilisée, a été déterminée au préalable [12] en enregistrant les spectres d'isotopes dont les intensités de transition sont bien connues $\left({ }^{207} \mathrm{Bi},{ }^{88} \mathrm{Y},{ }^{60} \mathrm{Co}\right)$.

Avec le dispositif de coïncidences $\gamma-\gamma$ lent-rapide, nous avons mesuré les spectres $\gamma$ en coïncidence avec 


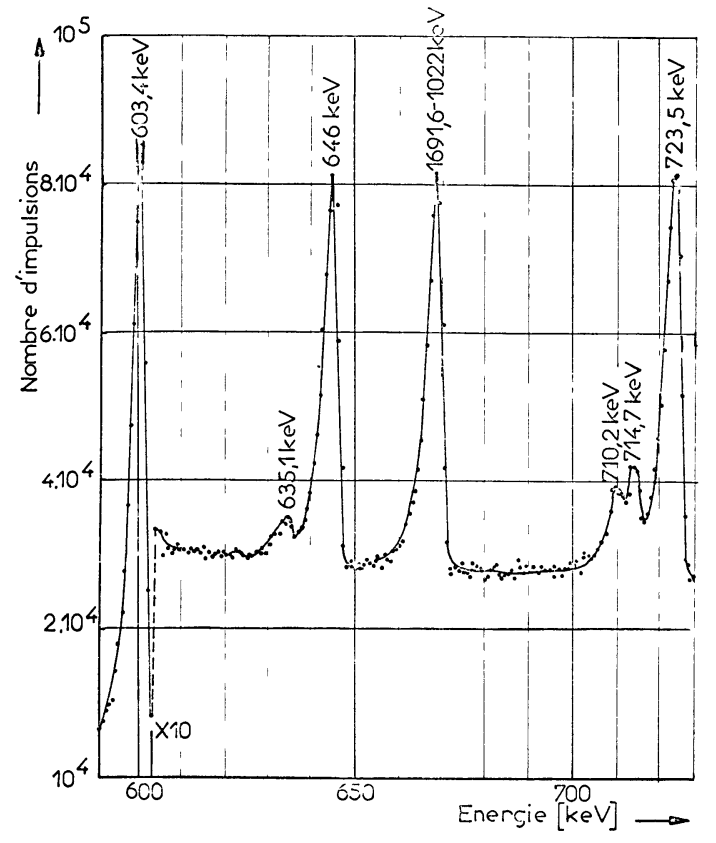

FIG. 1.

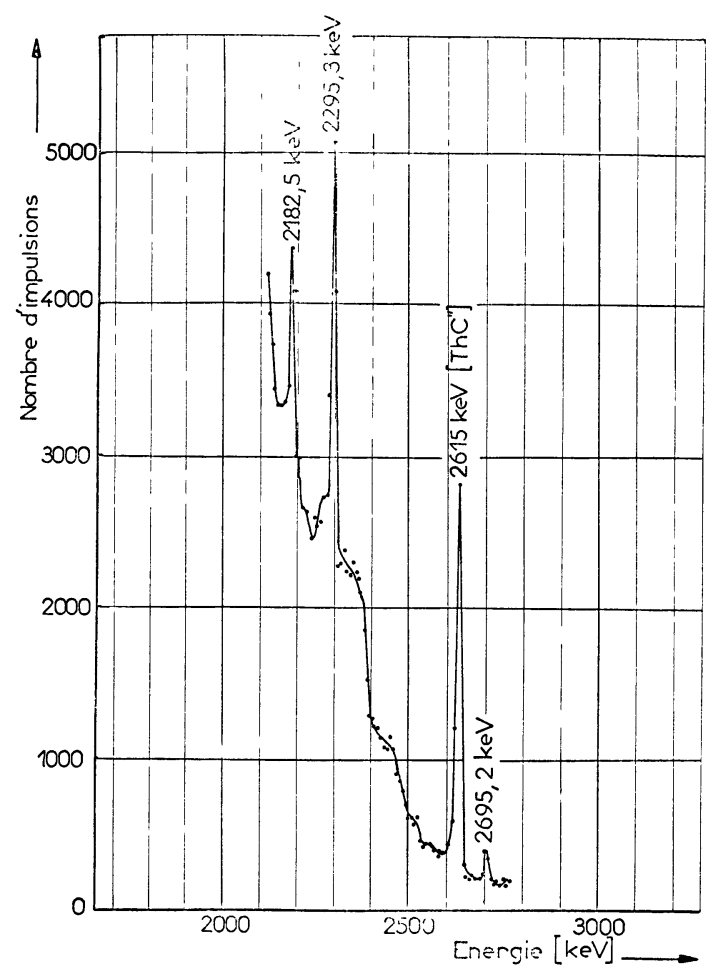

FIG. 3.

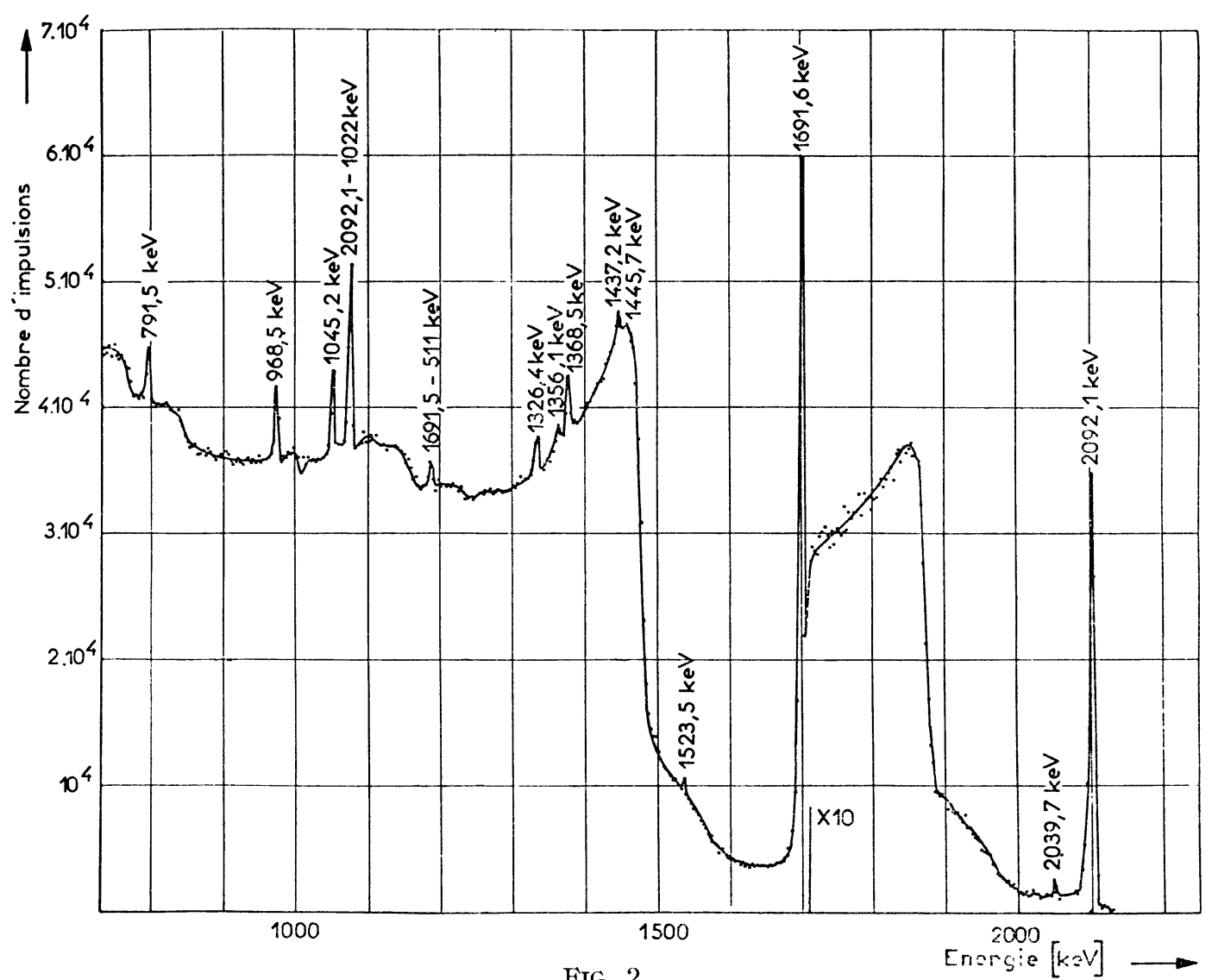

LE JOURNAl, DE PHYSIQUe. - T. 29. No9 2-3. FÉVRIER-MARS 1968 
TABLEAU I

ÉNERGie de TRANSITION (keV)

Travaux PRÉcÉdents

$602,8 \pm 0,8\left(^{\mathrm{a}}\right)$
632
$646 \pm 1\left(^{\mathrm{b}}\right)$
709
714
$723 \pm 1\left(^{\mathrm{b}}\right)$
789
970
1050
1320
1361
1370
1450
1500
1540
$1691,3 \pm 0,3\left(^{(}\right)$
2032
$2090,7 \pm 0,5\left(^{(}\right)$

Notre travail

$$
\begin{array}{r}
603,4 \pm 0,4 \\
635,1 \pm 0,7 \\
646,7 \pm 0,4 \\
710,2 \pm 0,4 \\
714,7 \pm 0,4 \\
723,5 \pm 0,4 \\
791,5 \pm 0,7 \\
968,5 \pm 0,7 \\
1045,2 \pm 0,7 \\
1326,4 \pm 0,7 \\
1356,1 \pm 0,7 \\
1368,5 \pm 0,7 \\
1437,2 \pm 0,7 \\
1445,7 \pm 0,7 \\
1523,5 \pm 0,9 \\
\\
1691,6 \pm 0,4 \\
2039,7 \pm 0,7 \\
2092,1 \pm 0,4 \\
2182,5 \pm 0,9 \\
2295,3 \pm 0,9 \\
2695,2 \pm 0,9
\end{array}
$$

INTENSITÉS RELATIVES $\gamma($ en \%) Travaux préGÉdents Notre travail

$\begin{array}{cc}- & - \\ 100 & 100 \\ 7,5 \pm 0,5\left(^{\mathrm{d}}\right) & 0,54 \pm 0,11 \\ & 7,41 \pm 0,44 \\ 3,7 & 2,9 \pm 0,10 \\ 15 \pm 1,5\left(^{\mathrm{e}}\right) & 3,2 \pm 0,13 \\ & 10,55 \pm 0,70 \\ 2 & 0,8 \pm 0,1 \\ 2 & 1,86 \pm 0,11 \\ 2 & 1,84 \pm 0,16 \\ 1 & 1,88 \pm 0,18 \\ 2,5 & 1,05 \pm 0,07 \\ 2 & 2,44 \pm 0,20 \\ & 1,76 \pm 0,18 \\ 1,7 & 1,06 \pm 0,20 \\ & 0,28 \pm 0,02 \\ 51 \pm 3 \text { (d) }^{\mathrm{d}} & \\ 6,3 & 46,08 \pm 3,3 \\ & 0,21 \pm 0,05 \\ & 5,87 \pm 0,09 \\ & 0,05 \pm 0,002 \\ & 0,08 \pm 0,003 \\ & 0,01 \pm 0,0005\end{array}$

(a) Réf. [18] ; (b) Réf. [4]; (c) Réf. [7] ; (d) Réf. [5] ; (e) Réf. [6].

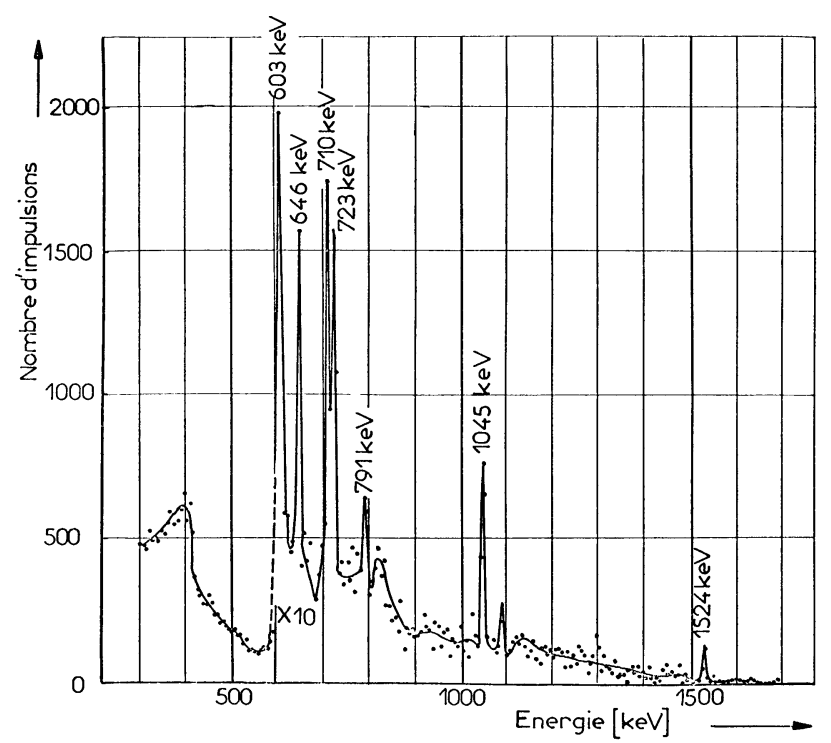

FIG. 4.

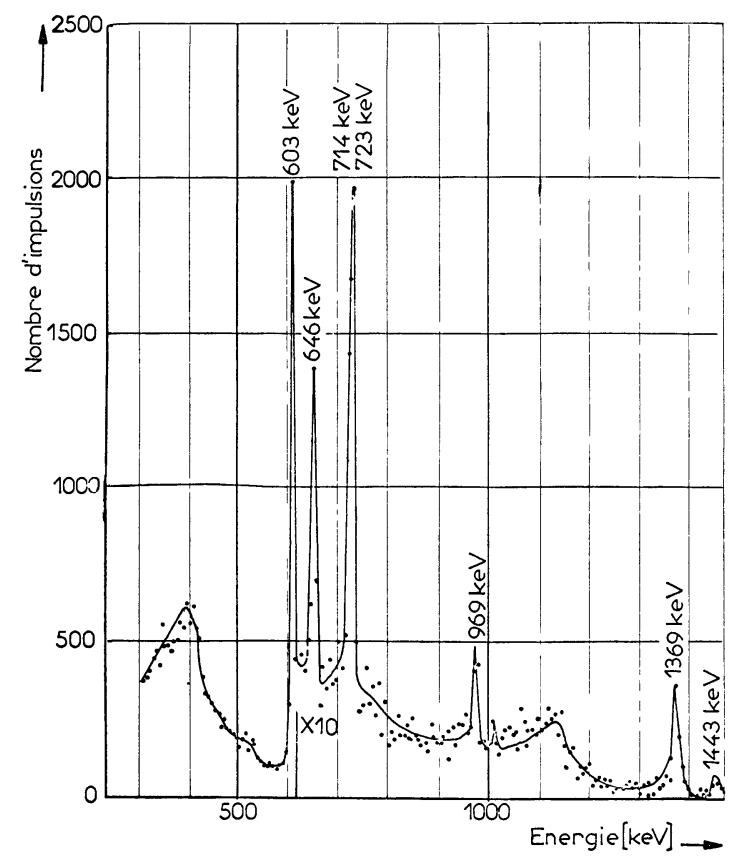

FIG. 5. 
les pics suivants : $646 \mathrm{keV}$ ( fig. 4) et $723 \mathrm{keV}$ ( fig. 5); la largeur des bandes d'énergie est d'environ $10 \mathrm{keV}$. Les résultats obtenus sont résumés dans le tableau II.

\section{TABLEAU II}

\begin{tabular}{cccccccc}
$\begin{array}{c}\text { ÉNERGIES } \\
(\mathrm{keV})\end{array}$ & \multicolumn{5}{c}{$\begin{array}{c}\text { TRANSITIONS } \gamma \text { EN COÏNCIDENCE } \\
(\mathrm{en} \mathrm{keV})\end{array}$} \\
- & \multicolumn{6}{c}{-} \\
646 & 603, & 646, & 710, & 723, & 791, & 1045, & 1523 \\
723 & 603, & 646, & 714, & 723, & 969, & 1369, & 1443
\end{tabular}

4. Schéma des niveaux de ${ }^{124}$ Te. - Le schéma de niveaux de ${ }^{124} \mathrm{Te}$ déduit des travaux antérieurs, ainsi que celui auquel on est conduit, compte tenu des nouveaux résultats acquis au cours de cette étude, est représenté sur la figure 6 . Le calcul précis des énergies a permis de modifier la place de plusieurs transitions : $710,2 \mathrm{keV}, 714,7 \mathrm{keV}, 968,5 \mathrm{keV}, 1445,7 \mathrm{keV}$, $1523,5 \mathrm{keV}$; un niveau supplémentaire a été introduit à $2773 \mathrm{keV}$, tandis que la présence d'un autre à $2041 \mathrm{keV}$ a été confirmée.

L'existence de ce dernier $(2041 \mathrm{keV})$ découle des coïncidences observées entre les rayonnements de 791,5 et $646,7 \mathrm{keV}$ d'une part, et 714 et $723 \mathrm{keV}$ d'autre part (tableau II). Ce niveau permet également de rendre compte des transitions de 2039,7 et $1437,2 \mathrm{keV}$ ( fig. 6). Aucune transition $\gamma$ n'existant en direction de ce niveau, son alimentation doit être attribuée à une composante provenant de ${ }^{124} \mathrm{Sb}$. Expérimentalement, un embranchement d'une intensité de 4 à $10 \%$ condui- sant à un niveau voisin de $2000 \mathrm{keV}$ (la précision de telles mesures ne dépasse guère $\pm 100 \mathrm{keV})$ a été signalé par différents auteurs [13]. Par suite, le spin de l'état fondamental de ${ }^{124} \mathrm{Sb}$ étant $3^{-}$, les règles de sélection des émissions $\beta$ permettent de limiter aux valeurs 2, 3 ou 4 le spin du niveau de $2041 \mathrm{keV}$. Par ailleurs, la présence d'une transition de $2039,7 \mathrm{keV}$ vers l'état fondamental $0^{+}$rend très improbable la valeur 4.

Le fait que la transition de $1523,5 \mathrm{keV}$ soit en coïncidence avec celle de $646,7 \mathrm{keV}$, mais non avec celle de 723,5 keV (tableau II), signifie qu'elle aboutit sur le niveau de $1250 \mathrm{keV}$, ce qui implique l'existence d'un état à $2773 \mathrm{keV}$, de spin 2, 3 ou 4 . Ce niveau est désexcité également par le rayonnement de $1445,7 \mathrm{keV}$ qui est en effet en coïncidence avec la raie de $723,5 \mathrm{keV}$ ( fig. 5).

Les niveaux à 2294,8 et $2695,3 \mathrm{keV}$ sont décrits généralement comme étant des états de vibration octopolaire $3^{-}$. Cette interprétation se trouve renforcée par notre observation des transitions vers le niveau fondamental.

Signalons enfin que nos résultats ne permettent pas de confirmer l'existence de niveaux à 1350 et $1900 \mathrm{keV}$ comme proposé par Girgis et Lieshout [6].

5. Conclusion. - On considère généralement que le noyau de ${ }^{124}$ Te est approximativement sphérique et que les niveaux d'excitation sont surtout dus à des vibrations de surface quadrupolaires. Le modèle de Bohr [14] prévoit ainsi un premier niveau excité $2^{+}$
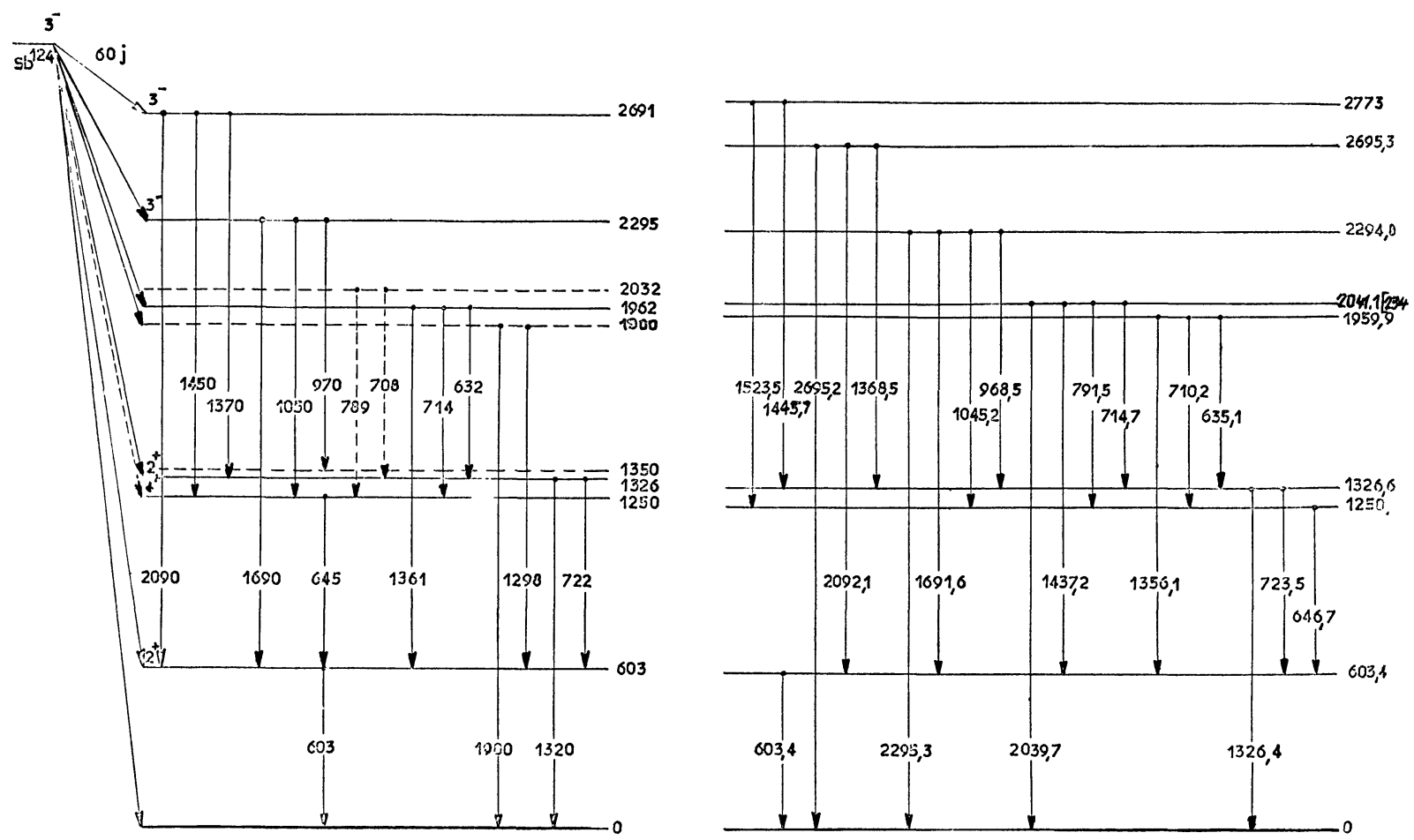

FIG. 6. 
et, à une énergie double, un triplet dégénéré $0^{+}, 2^{+}$, $4^{+}$. Par la suite, diverses variantes de ce modèle simple d'oscillation harmonique ont été développées, en particulier par Scharff-Goldhaber [1], Raz [2], Wilets et Jean [3]. Mais, comme l'ont montré Cookson et Darcey [9], aucune de ces théories ne permet de rendre compte du fait que le deuxième niveau $2^{+}$ de ${ }^{124} \mathrm{Te}$ soit situé au-dessous du niveau $4^{+}$, contrairement à la situation qui prévaut dans les noyaux voisins, le cadmium par exemple.

Le modèle général du rotateur asymétrique a été appliqué aux noyaux pair-pair par Mallmann [15]. L'accord des précisions théoriques avec les résultats expérimentaux est satisfaisant dans de nombreux noyaux [15-16]. Néanmoins, ce modèle ne semble pas pouvoir rendre compte des propriétés des isotopes ayant des nombres de protons ou de neutrons proches des nombres magiques ( $\left.{ }^{1}\right)$. Dans le cas de ${ }^{124} \mathrm{Te}$, en admettant que les énergies, spins et parités sont ceux indiqués sur la figure 6 , le calcul de Mallmann prévoit des états $3^{+}$et $4^{+}$respectivement à 1665 et $2046 \mathrm{keV}$. Un niveau à $1657 \mathrm{keV}$ a été mis en évidence par Cookson et Darcey par diffusion inélastique de protons, mais son spin n'a pas pu être déterminé [9]. Par ailleurs, le niveau à $2041 \mathrm{keV}$ dont nous avons montré l'existence a vraisemblablement un spin inférieur à 4 et ne correspond pas alors à l'état $4^{+}$prévu.

(1) Selon Suarez et Aisenberg [16], le modèle de Mallmann s'applique aux noyaux pour lesquels :

$$
\left|Z-Z_{m}\right| \leqslant 0,08 Z_{m} \text { ou }\left|N-N_{m}\right| \leqslant 0,08 N_{m}
$$

où $Z_{m}$ et $N_{m}$ désignent les nombres magiques de protons et de neutrons respectivement.

\section{BIBLIOGRAPHIE}

[1] SCharFF-Goldhaber (G.) et Weneser (J.), Phys. Rev., 1955, 98, 212.

[2] Raz (B. J.), Phys. Rev., 1959, 114, 1116.

[3] Wilets (L.) et Jean (M.), Phys. Rev., 1956, 102, 788.

[4] Zolotavin (A. V.), Grigoryev (E.) et Abroyan (M. A.), Izv. Akad. Nauk, Ser. Fiz., 1956, 20, 289.

[5] DZhelepov (B. S.) et Zhukovskit (N. N.), Nucl. Physics, 1958, 6, 655.

[6] Girgis (R. K.) et Van Lieshout (R.), Physica, 1959, 25, 133.

[7] Marlow (K. W.), Nucl. Physics, 1964, 51, 684.

[8] Dorikens-Vanpraeit (L.), Demuynck (J.) et DoriKENS (M.), Nucl. Phys., 1965, 73, 539.

[9] Cookson (J. A.) et DARCEY (W.), Nucl. Physics, 1965, 62, 326 .

[10] KIM (Y. S.) et CoHen (B. L.), Phys. Rev., 1966, 142, 788 .
[11] Groshev (L. V.), Demidov (A. M.) et Shadiev (N.), Yadernaya Fizika, 1966, 4, 238.

[12] Stab (L.), Henck (R.), SIFFERT (P.) et Coche (A.), Nucl. Instr. Methods, 1965, 35, 113.

[13] Dzhelepov (B. S.) et Peker (L. K.), "Decay Schemes of Radioactive Nuclei ", Pergamon Press, 1961.

[14] Bohr (A.), Mat. Fys. Medd. Dan. Vid. Selsk, 1952, 26, 14

[15] Malimann (C. A.), Nucl. Physics, 1961, 24, 535.

[16] SuAREZ (J. F.) et DE AISEnBERG (E. Y.), Nucl. Physics, 1967, A 90, 449.

[17] Beliaev (S. T.) et Zelevinsky (V. G.), Nucl. Physics, 1962, 39, 582.

[18] Robinson (R. L.), STELSON (P. H.), MCGowaN (F. K.), FORD (J. L. C.) et MIINER (W. T.), Nucl. Physics, 1964, 74, 281. 